\title{
Neural network modelling of Abbott-Firestone roughness parameters in honing processes
}

\begin{abstract}
Author
Address

Abstract:

In present study, three roughness parameters defined in the Abbott-Firestone or bearing area curve, Rk, Rpk and Rvk, were modeled for the rough honing processes by means of Artificial Neural Networks (ANN). Input variables were grain size and density of abrasive, pressure of abrasive stones on the workpiece's surface, tangential or rotation speed of the workpiece and linear speed of the honing head. A back propagation algorithm was used for training the networks. Two strategies were considered, use of either one network for modeling the three parameters at the same time and use of three networks, one for each parameter. Best network was chosen among different structures, having either one or two hidden layers. When one network is considered, best solution corresponds to two hidden layers having 26 and 11 neurons. However, overall best neural network consists of three networks, one for each roughness parameter, with one hidden layer having 25,9 and 5 neurons respectively.
\end{abstract}

Keywords: Roughness; honing; artificial neural networks; neurons; back propagation algorithm; grain size; density of abrasive; linear speed; tangential speed; pressure.

Reference to this paper should be made as follows: Author. (xxxx) 'Title', Int. J. $x x x x x x x x x x x x$ xxxxxxxxxxxx,

Biographical notes:

\section{Introduction}

Surface finish of combustion engine cylinders is directly related to friction, wear and oil consumption (Tung \& McMillan 2004). Final honing process performed to internal surface of cylinders provides a crosshatch pattern that favours oil flowing. AbbottFirestone related parameters Rk-core roughness, Rpk- reduced peak height and Rvkreduced valley depth, defined in standard ISO 13565-2 (ISO 1996), are commonly employed for characterizing surface finish after honing operations (Deepak Lawrence et al. 2014). As a general trend, Rk value is recommended to be higher than Rpk and Rvk, in order to assure wear resistance of the surface. Next, Rvk should be higher than Rpk, so as to improve lubricant retention and oil efficiency. Thus, Rpk value is expected to be reduced after the running in period of an engine (Feng et al. 2002). It is possible to reduce 
Rpk parameter from the manufacturing process by means of plateau-honing operation, in which a very fine grain size is employed to remove peaks from previous honing operations (Kanthababu et al. 2010; Lawrence \& Ramamoorthy 2016). The plateau honing operation allows controlling bearing area at the top of the surface.

Roughness achieved in honing processes depends on many parameters related to process kinematic, structure and composition of honing stones and lubrication oil among others (Bell et al. 1981; Feng et al. 2015). For this reason, analytical models are discarded. Some authors developed numerical models for defining surface obtained in honing processes (Voronov et al. 2009), while both regression and artificial neural networks (ANN) models are recommended in this case. As for ANN, for example, surface roughness and tool wear in turning processes were predicted by (Özel \& Karpat 2005), as a function of edge geometry, hardness and cutting conditions. They used the backpropagation algorithm. In milling operations, (Benardos \& Vosniakos 2002) employed the Levenberg-Marquardt algorithm to implement the backpropagation training, in order to model surface roughness as a function of cutting conditions and cutting tool wear among other factors. (Chavoshi 2013) used neural networks to predict roughness to be obtained in face milling. Regarding abrasive machining processes, for example, (Liao \& Chen 1994) predicted average roughness Ra by means of two layer neural networks. They considered bond type, mesh size, concentration, work speed and depth of cut as inputs. (Petri et al. 1998). developed a neural network model for predicting surface finish and dimensional change as a function of workpiece parameters, media characteristics, machining parameters and technical specifications. (Teimouri \& Baseri 2013) used ANN and neuro-fuzzy interference system to predict surface roughness in magnetic abrasive finishing processes, as a function of parameters such as voltage, mesh size and rotation speed. (Ben Fredj et al. 2002) simulated average roughness Ra and peak-to-valley roughness Rt as a function of process parameters such as table speed, depth of cut, grain size, dressing depth, number of passes and workpiece material in grinding processes. They employed one layer networks. (Deng et al. 2009) optimized surface roughness in a camshaft grinding operation as a function of different cutting conditions. They combined neural networks having one hidden layer with genetic algorithm. (Maksoud et al. 2003) employed neural networks for both designing the grinding process and taking a decision about accepting the process, redesigning it or dressing the grinding wheel. (Aguiar et al. 2008) used three layer networks for predicting average roughness $\mathrm{Ra}$ as a function of acoustic emission and cutting power in grinding processes. As for honing processes, only few studies are known about roughness prediction by means of ANN. For example, (Feng et al. 2002) modelled roughness parameters defined in the Abbott-Firestone curve for both rough and finish honing operations. They considered variables such as grit size, honing time and pressure. Later, they compared the use of networks with different cross-validation methodologies and different number of hidden layers (Feng et al. 2005). (Pu et al. 2010). optimized the honing process as a function of stone granularity, stroke length, stroke speed and spindle speed. They used the Levenberg-Marquardt function in a backpropagation model. (Shaikh et al. 2015) used ANN for modelling surface roughness and material removal rate as a function of process parameters in electrochemical honing, However, other parameters such as density of abrasive and speed of honing stone are also known to influence roughness in honing processes (Buj-Corral et al. 2014). Moreover, in industry there is a strong demand for tools that allow predicting roughness without the need to 
carry out a great amount of experimental tests before starting production of a certain workpiece.

In a previous paper by the authors of the present paper, an indirect model for roughness was presented (Sivatte-Adroer et al. 2016). Main objective of this paper is to select best ANN configuration for modelling three Abbott-Firestone roughness parameters Rk, Rpk and Rvk as a function of five different variables of the honing process: grain size and density of abrasive, pressure, linear speed and tangential speed. For doing this, experimental tests were performed. A multilayer perceptron trained by means of the back propagation algorithm was used. From obtained results, best neural network configuration was selected among different ones considering one or two hidden layers. Two different strategies were considered, consisting of use of either one network for the three roughness parameters considered, or use of three different networks, one for each roughness parameter. Experimental data from design of experiments were used to train and validate the networks. Additional experiments were used as test data for comparing different models.

Models presented in this paper will help users of honing machines to select most appropriate process parameters values that are necessary achieve required roughness values. It will not be necessary to perform a high number of experimental trial and error tests, which are cost and time consuming. Models will also be useful for investigating the effect of each process parameter separately on surface roughness.

\section{Materials and methods}

\subsection{Honing tests}

In the interior honing process a honing head provided with abrasive stones removes material from the internal surface of a cylinder. In industrial machines, usually the honing head has a reciprocating movement combined with a rotation movement. Figure 1 shows a schematic drawing of cylinders with tangential speed VT, linear speed VL and cutting speed VC. Honing angle $\alpha$ is defined by tangential and linear speed. 
Author

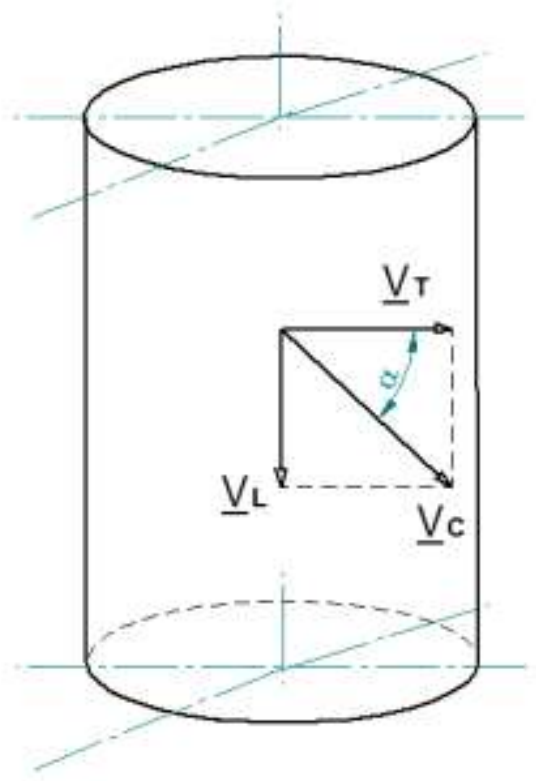

Figure 1 Schematic drawing of the honing process

In the present paper, honing tests were performed in a horizontal Honingtec test machine. In this case, tangential speed is obtained by means of rotation of the workpiece, while the honing head has linear alternate movement (Figure 2). The honing head is provided with three abrasive stones. The machine was specially designed for monitoring different process variables such as pressure, linear and tangential speed, workpiece diameter, etc. 


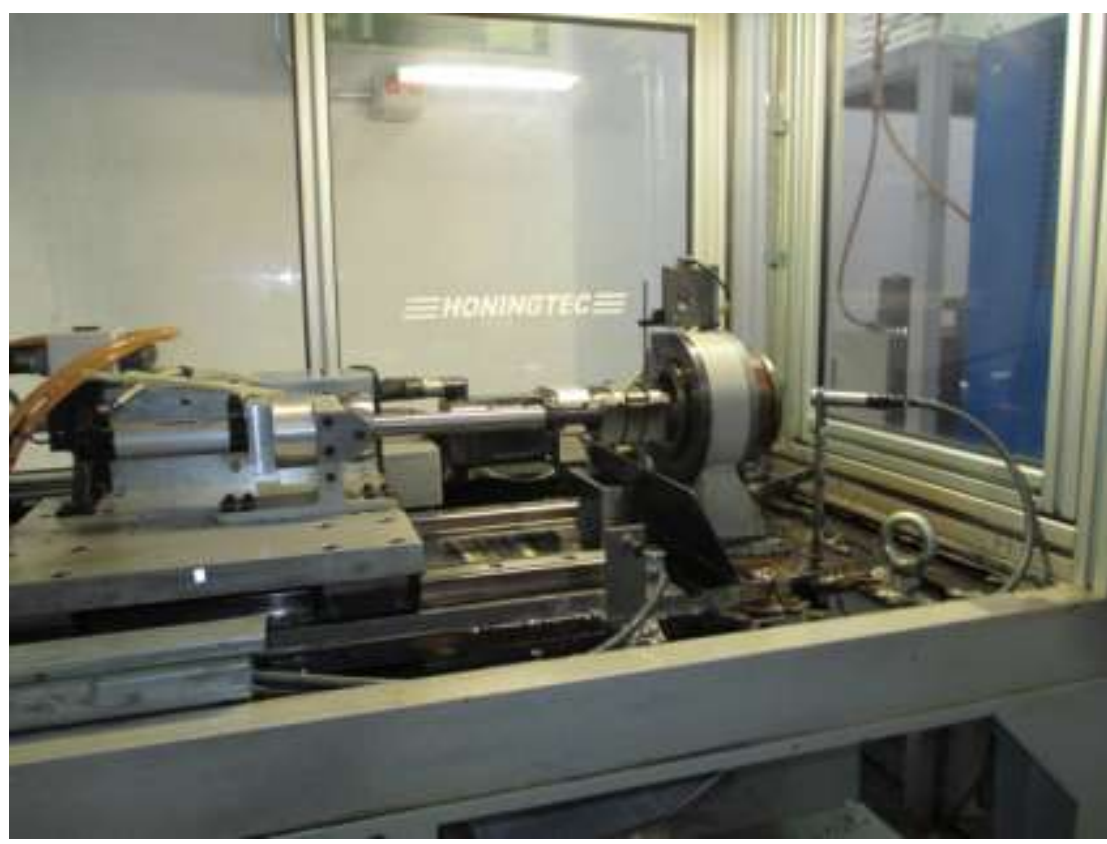

Figure 2 Honing test machine.

Steel St-52 cold-drawn seamless tubes of dimensions $300 \mathrm{~mm}$ in length, $90 \mathrm{~mm}$ external diameter and $80 \mathrm{~mm}$ internal diameter were honed. Abrasive stones with metallic bond and cubic boron nitride (CBN) abrasive were used.

Process variables considered were linear speed of the honing head VL ( $\mathrm{m} / \mathrm{min})$, tangential speed of the workpiece VT $(\mathrm{m} / \mathrm{min})$, pressure with which honing stones are held against the workpiece PR $\left(\mathrm{N} / \mathrm{cm}^{2}\right)$ and features of the honing stones such as grain size of abrasive GS, according to (FEPA 1997), and density of abrasive DE, defined in (ISO 2005) [16]. GS number has a correspondence with grit range in $\mu \mathrm{m}$. DE is defined by a number which is related to weight of abrasive in carats over volume of abrasive stone in $\mathrm{cm}^{3}$ ( 1 carat $\left.=0.2 \mathrm{~g}\right)$. For example, number 100 indicates 4.4 carats of abrasive $/ \mathrm{cm}^{3}$. Other quantities of abrasive can be found by following a linear relationship.

Selected responses were three parameters from the Abbott-Firestone or bearing curve, Rk-core roughness, Rpk- reduced peak height and Rvk- reduced valley depth. Experimental tests were defined by means of a fractional factorial model design $2^{5-1}$, with one central point and ten face centered points, with a total amount of 27 experiments. They correspond to experiments 1 to 27 in Table 1. Data samples collected from experiments from the fractional factorial design were randomly divided into two groups: 22 samples $(80 \%)$ were used for training the networks and 5 samples $(20 \%)$, for validating them. Additional 6 test samples were employed for comparing the different models. They were numbered 28 to 33 in Table 1 . Table 1 summarizes honing conditions as well as roughness results. 
Author

Table 1 Experiments performed and roughness Rk, Rpk and Rvk values

\begin{tabular}{|c|c|c|c|c|c|c|c|c|}
\hline Experiment & $\begin{array}{c}\text { GS } \\
\text { (FEPA) }\end{array}$ & $\begin{array}{c}\text { DE } \\
\text { (ISO6104) }\end{array}$ & $\begin{array}{c}\mathrm{PR} \\
\left(\mathrm{N} / \mathrm{cm}^{2}\right)\end{array}$ & $\begin{array}{c}\mathrm{VT} \\
(\mathrm{m} / \mathrm{min})\end{array}$ & $\begin{array}{c}\mathrm{VL} \\
(\mathrm{m} / \mathrm{min})\end{array}$ & $\begin{array}{c}\mathrm{Rk} \\
(\mu \mathrm{m})\end{array}$ & $\operatorname{Rpk}(\mu \mathrm{m})$ & $\operatorname{Rvk}(\mu \mathrm{m})$ \\
\hline 1 & 136 & 45 & 550 & 50 & 30 & 10.38 & 2.85 & 1.42 \\
\hline 2 & 136 & 45 & 550 & 40 & 30 & 10.67 & 2.74 & 1.75 \\
\hline 3 & 136 & 45 & 550 & 40 & 20 & 7.52 & 2.67 & 1.82 \\
\hline 4 & 181 & 45 & 550 & 40 & 30 & 12.45 & 3.22 & 2.64 \\
\hline 5 & 181 & 60 & 700 & 50 & 40 & 14.51 & 6.35 & 5.32 \\
\hline 6 & 91 & 30 & 400 & 50 & 20 & 6.46 & 2.07 & 1.82 \\
\hline 7 & 91 & 60 & 400 & 50 & 40 & 3.97 & 2.40 & 2.24 \\
\hline 8 & 181 & 60 & 400 & 30 & 40 & 15.71 & 5.40 & 4.03 \\
\hline 9 & 91 & 30 & 400 & 30 & 40 & 6.55 & 2.42 & 1.51 \\
\hline 10 & 91 & 60 & 400 & 30 & 20 & 6.18 & 3.06 & 1.64 \\
\hline 11 & 91 & 30 & 700 & 50 & 40 & 10.17 & 2.52 & 0.58 \\
\hline 12 & 136 & 45 & 550 & 40 & 40 & 11.04 & 3.83 & 1.66 \\
\hline 13 & 91 & 30 & 700 & 30 & 20 & 8.75 & 2.65 & 0.98 \\
\hline 14 & 91 & 45 & 550 & 40 & 30 & 8.57 & 2.48 & 1.39 \\
\hline 15 & 181 & 30 & 400 & 30 & 20 & 8.47 & 4.56 & 3.27 \\
\hline 16 & 136 & 60 & 550 & 40 & 30 & 11.21 & 4.33 & 2.53 \\
\hline 17 & 136 & 45 & 550 & 30 & 30 & 9.63 & 2.46 & 2.02 \\
\hline 18 & 181 & 30 & 400 & 50 & 40 & 14.78 & 4.80 & 1.76 \\
\hline 19 & 91 & 60 & 700 & 50 & 20 & 5.72 & 2.60 & 2.31 \\
\hline 20 & 181 & 30 & 700 & 50 & 20 & 9.42 & 3.65 & 3.65 \\
\hline 21 & 136 & 45 & 400 & 40 & 30 & 7.28 & 2.53 & 2.17 \\
\hline 22 & 136 & 45 & 700 & 40 & 30 & 9.21 & 2.83 & 2.55 \\
\hline 23 & 181 & 60 & 700 & 30 & 20 & 18.43 & 6.46 & 4.73 \\
\hline 24 & 181 & 30 & 700 & 30 & 40 & 14.05 & 3.71 & 1.70 \\
\hline 25 & 91 & 60 & 700 & 30 & 40 & 7.25 & 2.35 & 0.99 \\
\hline
\end{tabular}




\begin{tabular}{r|r|r|r|r|r|r|r|r}
\hline 26 & 136 & 30 & 550 & 40 & 30 & 9.88 & 2.72 & 1.87 \\
\hline 27 & 181 & 60 & 400 & 50 & 20 & 15.55 & 5.48 & 5.02 \\
\hline 28 & 151 & 35 & 625 & 45 & 25 & 7.20 & 3.16 & 3.61 \\
\hline 29 & 181 & 45 & 534 & 46 & 40 & 15.01 & 7.04 & 3.64 \\
\hline 30 & 91 & 45 & 624 & 39 & 28 & 4.83 & 1.89 & 2.07 \\
\hline 31 & 136 & 45 & 542 & 44 & 28 & 7.00 & 3.68 & 2.93 \\
\hline 32 & 181 & 60 & 581 & 39 & 43 & 11.36 & 5.69 & 4.85 \\
\hline 33 & 136 & 45 & 545 & 43 & 25 & 6.41 & 4.04 & 2.92 \\
\hline
\end{tabular}

\subsection{Roughness and cylindricity measurements}

Roughness measurement was conducted with a Taylor Hobson Talysurf 2 roughness meter with $\mu$ ltra software (v. 4.6.8). According to a previous study, for each cylinder 9 roughness measurements were taken, which were separated $40^{\circ}$ along a diametric circumference on the internal surface of the cylinder at $50 \mathrm{~mm}$ from its end (Buj-Corral \& Vivancos-Calvet 2011). Average value of the nine measurements was taken into account for each cylinder, for each roughness parameter considered, Rk, Rpk and Rvk. . A Gaussian filter was used with high pass cut-off and sampling length of $0.8 \mathrm{~mm}$, low pass cut-off of $0.025 \mathrm{~mm}$ and evaluation length of $4 \mathrm{~mm}$.

Cylindricity was measured by means of a Taylor Hobson Talyrond 252 roundness meter, with Gaussian filter with roundness cutoff of 50 upr.

\subsection{Neural networks}

Networks addressed the direct problem, in which roughness parameters are predicted as a function of process variables. Two different strategies were taken into account for defining the networks. First one consists of one network with the five process variables as inputs and the three roughness parameters as outputs (Figure 3a). Second one consists of considering three separate networks, one for each roughness parameter as an output (Figure 3b). 
Author

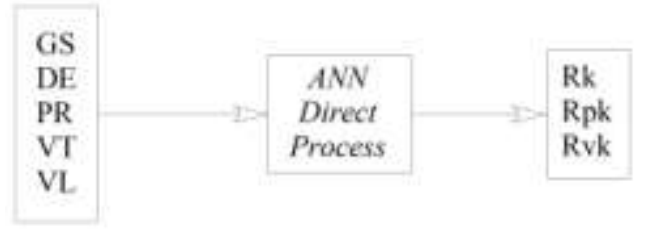

(a)

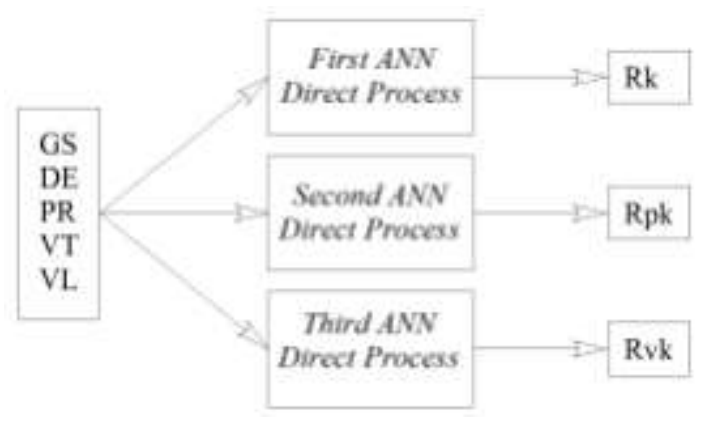

(b)

Figure 3 Flow chart for the two different strategies: a) one network, b) three networks.

Neural networks were modelled by means of Matlab 7.8.0 347, with a specific program. Different configurations were tested, based on feed forward multilayer perceptron and a back propagation supervised training algorithm with cross validation system. In the present paper, two different perceptron configurations were considered, first one with one hidden layer and second one with two hidden layers. It was discarded to use higher number of layers in order to avoid overtraining, which means memorizing training data rather than training the network. Networks were configured with five input variables, corresponding to the five process parameters GR, DE, PR, VT and VL, either one or two hidden layers with sigmoidal function, and an output layer with linear purelin function. The five input variables were normalized so as to avoid some of them to have more weight than the rest of the variables in the model. Output variables were roughness parameters Rk, Rpk and Rvk. It was not necessary to normalize responses, since they have similar values and units. As an example, Figure 4 shows the structure of one network with five input variables, one hidden layer with sigmoidal function, one output layer with purelin function and three output parameters. 


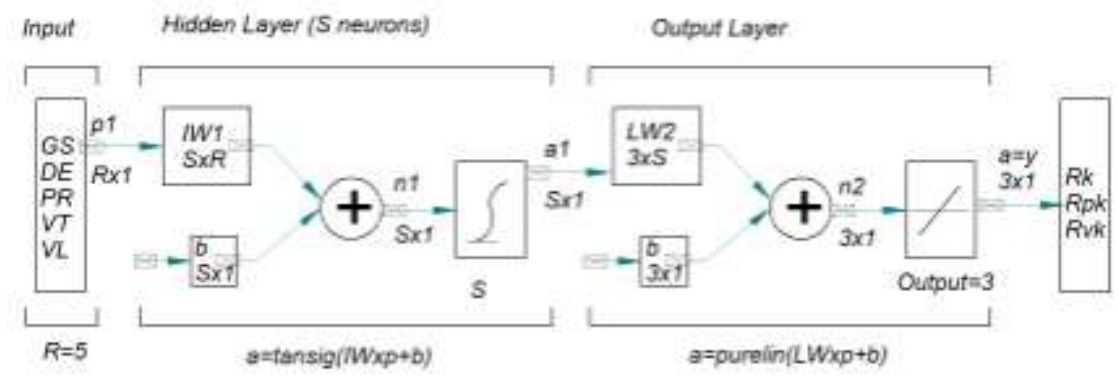

Figure 4 Schematic drawing of one network with one hidden layer.

Training data were used for training the networks in an iterative way by modification of the weight matrices IW1 and LW2 (Figure 4). Training was stopped when reduction in quadratic average error obtained with validation data was lower than a certain previously defined value, $1.00 \mathrm{e}^{-10}$. Test data were used to evaluate and compare performance of the different neural models.

One layer networks were trained with number of neurons ranging from 4 to 100 . For two hidden layers, total number of neurons tested was $\mathrm{N}+50 \%$, where $\mathrm{N}$ is best number of neurons selected for one hidden layer. First, 2/3 of total number of neurons was assigned to the first layer and $1 / 3$ of total number of neurons was assigned to the second layer. Later, variations of \pm 1 neurons in each layer were considered, so that summation of neurons was constant. The perceptron configuration that better models validation data was searched. Thus, best network was selected as the one that minimizes root mean squared error (mse) of validation data (Equation 1).

$$
\text { mse }=\sqrt{\sum_{i=1}^{n} \frac{\left(t_{i}-y_{i}\right)^{2}}{n}}
$$

where $t_{i}$ denotes the experimental value of variables $\mathrm{Rk}$, Rpk or Rvk corresponding to variables of the 'ith' experiment, $y_{i}$ is the simulated value of variables Rk, Rpk and Rvk for the 'ith' process, and $n$ is number of experiments considered. Root mean squared error has the same units as the magnitude being considered.

\section{Results}

\subsection{Roughness and cylindricity}

Experimental Rk, Rpk and Rvk values, corresponding to average of two replicates, are presented in Table 1 for the 33 experiments performed. As a general trend, highest Rpk, Rk and Rvk values correspond to high grain size of 181 and high density of 60 , for example in experiments 5, 8, 23 and 27. It is well known that grain size of abrasive is directly related to roughness (Bell et al. 1981). The higher grain size of abrasive, the broader honing marks are and the higher roughness is. As for density, a higher proportion of grains will help cutting operation, although an excessively high density could lead to difficulties in cutting due to lack of space to accommodate chip, and subsequent clogging of the stones. On the other hand, lowest Rpk, Rk and Rvk values were obtained when 
using low grain size, regardless of density employed (experiments $6,7,9,10,11,13,14$, 19 and 25). This suggests that, for rough operations performed with high grain size, influence of density is important. However, for semifinish operations performed with lower grain size, lower quantity of material is removed and clogging is not so likely to appear.

Highest Rk, Rpk and Rvk value corresponds to high grain size combined with high density, high pressure and low linear and tangential speed (experiment 23). Experiment 5, which was performed with high grain size, high density, high pressure and high linear and tangential speed also provided high roughness values. However, high roughness values can be also achieved with low pressure values, as shown in experiments 8,18 and 27. This suggests that, within the range studied, effect of pressure is not so important as effect of grain size and density of abrasive.

Very low effect was found in this case between tangential speed VT and roughness parameters Rk, Rpk or Rvk, as well as for linear speed VL and the same roughness parameters.

Cylindricity error measured values ranged between $12.0 \mu \mathrm{m}$ and $41.8 \mu \mathrm{m}$. Similar cylindricity values up to $36.7 \mu \mathrm{m}$ were reported by Lei et al(Lei et al. 2011).

As an example, roughness profiles are shown in Figures $5 \mathrm{a}$ and $5 \mathrm{~b}$ for experiments 23 and 9 respectively.

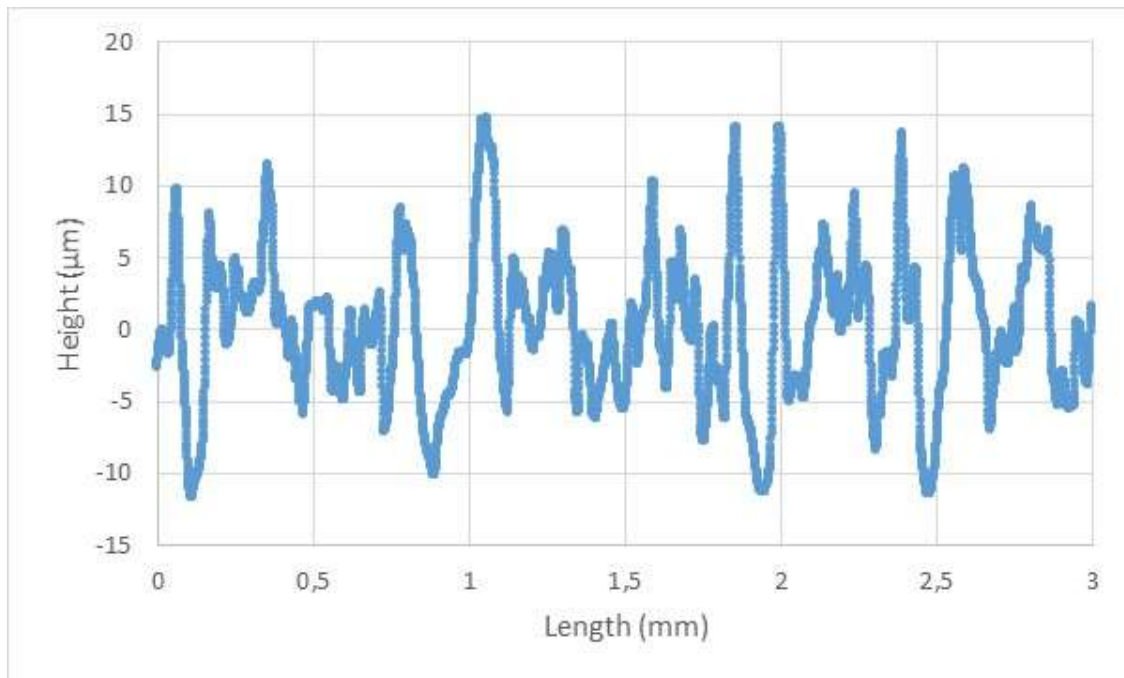




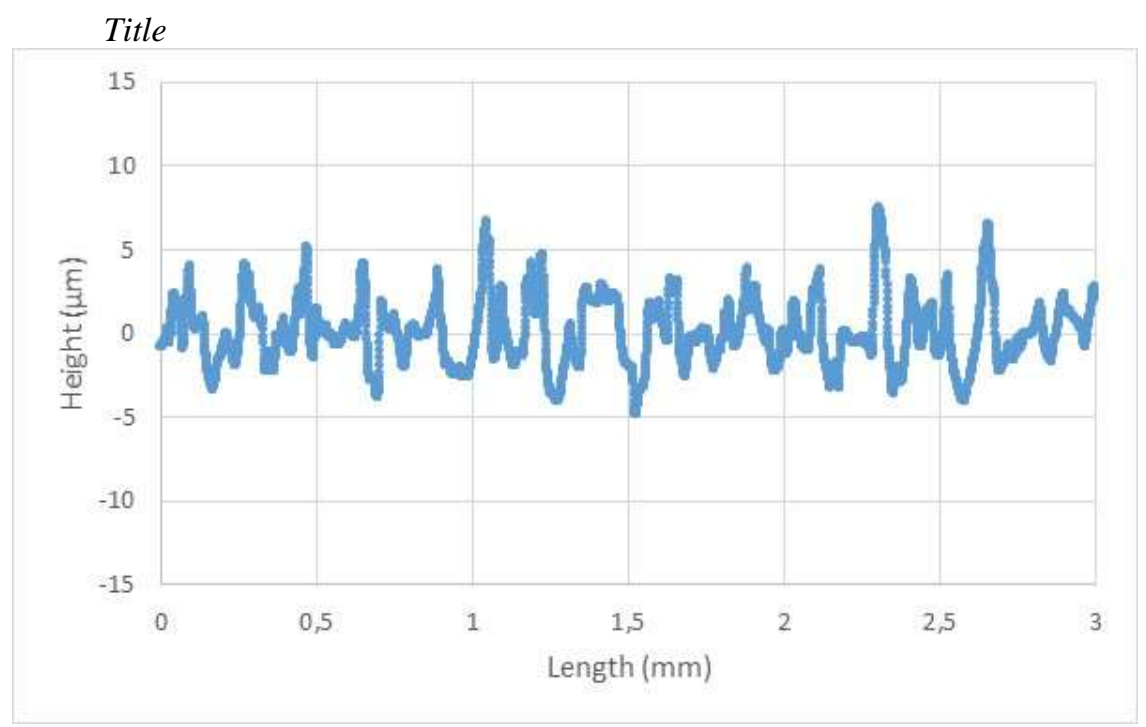

(b)

Figure 5. Roughness profiles for: a) Experiment 23, b) Experiment 9.

Irregular profiles were obtained in both cases, which are usual in abrasive machining processes. Higher peaks and valleys are observed for high grain size, density and pressure (Figure 5a) than for low grain size, density and pressure (Figure 5b).

\subsection{One neural network model}

For one neural network and one hidden layer, number of neurons was tested between 4 and 100. Average value for the three roughness parameters of mse for the validation experiments is shown in Fig. 6 for different number of neurons. 
Author

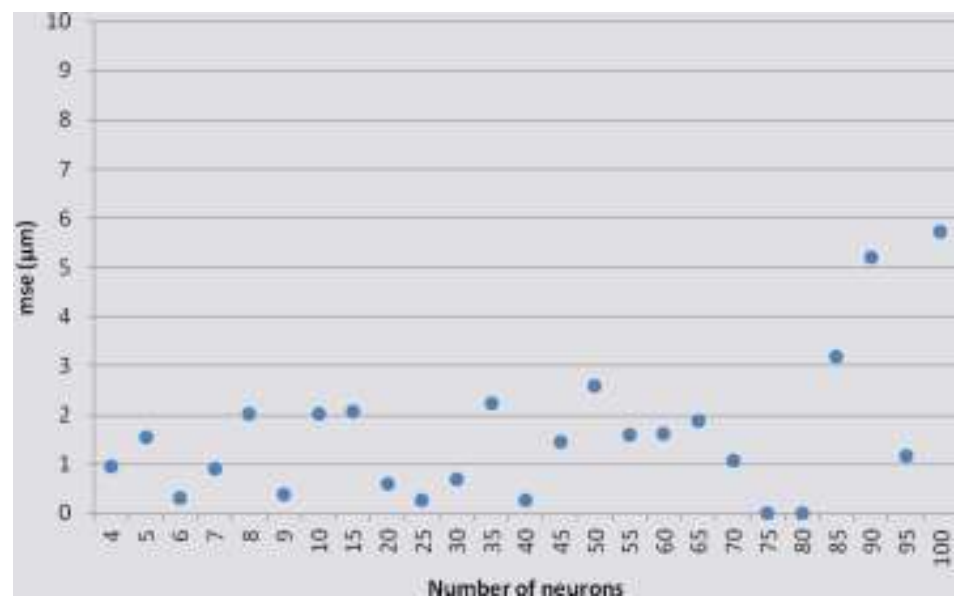

Figure 6. Average mse of validation experiments vs. number of neurons, for one network and one hidden layer.

For one network and one hidden layer, a first analysis recommends that use of number of neurons of 85 or higher be discarded, since especially high mse values for validation data, up to almost 6 were found in many cases. Lowest mse values for the validation data correspond to 75 and 80 neurons. However, such mse values are very close to zero, suggesting that the networks have memorized data instead of learning during training process. In order to check this, models having lowest mse of validation data were compared. For doing this, test data were employed. Model with 25 neurons was selected, since average mse value for test experiments was lowest among all models studied.

Taking into account 25 neurons as a start point for one layer, different number of neurons was tested for two layer networks: 20-17, 21-16, 22-15, 23-14, 24-13, 25-12, 26-11, 27 $10,28-9,29-8$ and 30-7, according to explanation in Section 2. Results are presented in Figure 7. 


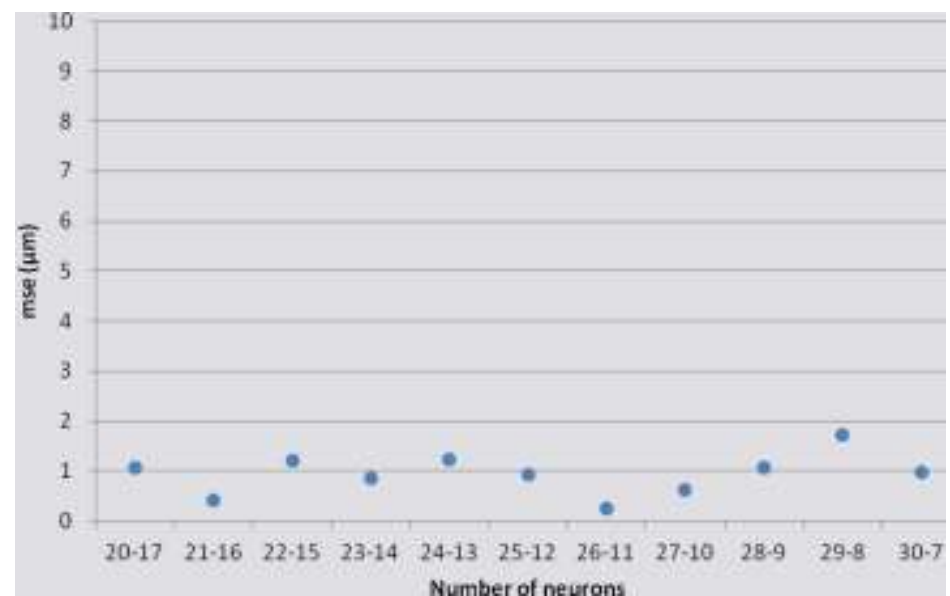

Figure 7. Average mse of validation experiments vs. number of neurons, for one network and two hidden layers

For one network and two hidden layers, low mse values below 2 were found for validation data in all cases studied. Thus, highest mse values were higher for one layer than for two layers, when one network was considered. For two layers, best network, having lowest mse value of validation experiments, corresponds to 26 and 11 neurons in the first and second layer respectively.

In order to compare the model with one hidden layer having 25 neurons, with the model with two hidden layers with 26 and 11 neurons respectively, test experiments were employed. Results are presented in Table 2.

Table 2 Comparison between models for one network.

\begin{tabular}{c|c|c}
\hline $\begin{array}{c}\text { Number } \\
\text { of layers }\end{array}$ & $\begin{array}{c}\text { Number of } \\
\text { neurons }\end{array}$ & mse \\
\hline One layer & 25 & 2.335 \\
\hline $\begin{array}{c}\text { Two } \\
\text { layers }\end{array}$ & $26-11$ & 1.997 \\
\hline
\end{tabular}

Best option for the strategy of using one network is use of two hidden layers with 26 and 11 neurons respectively, with lowest mse value of test experiments of 1.997 .

\subsection{Three neural network model}

For three neural networks and for one hidden layer, different number of neurons was tested between 4 and 100, for each one of the considered roughness parameters. As an example, mse values for validation data are presented for Rk in Figure 8. 


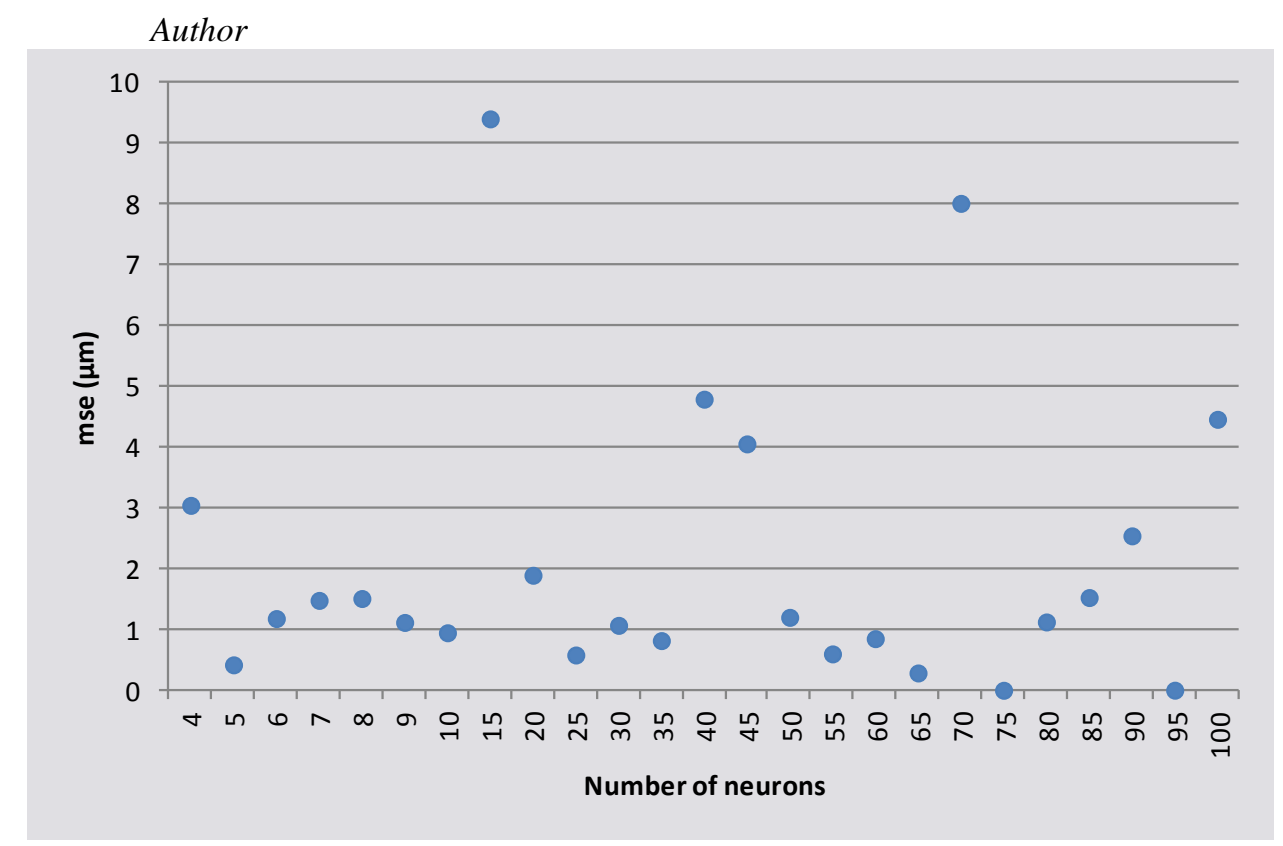

Figure 8. Mse of validation experiments vs. number of neurons, for Rk (three networks) and one hidden layer.

For parameter Rk, very high mse values were reported for 15 and 70 neurons. For example, for 15 neurons mse value was higher than 9 . Such values are much higher than those found for one network models, which were below 6 in all cases (Figure 3). On the contrary, extremely low mse values for 75 and 95 neurons suggest that the network memorized data during training step. For this reason, models having lowest mse value for validation data were compared. Model having 25 neurons was selected, since it showed lowest mse value for test experiments. A similar procedure was followed for parameters Rpk and Rvk, and selected number of neurons was 9 and 5 respectively. For Rpk and Rvk, when one layer was considered, mse values for validation data did not exceed 6 . When two layers were considered, quite lower mse values for validation data were reported, below 3 in all cases. Thus, highest mse values are higher for one than for two layers when three different networks are considered.

Regarding two layer networks, as an example, for parameter Rk different configurations were tested with 25 neurons as a start point, having 20-17, 21-16, 22-15, 23-14, 24-13, $25-12,26-11,27-10,28-9,29-8$ and 30-7 neurons respectively. Results are shown in Figure 9 . 


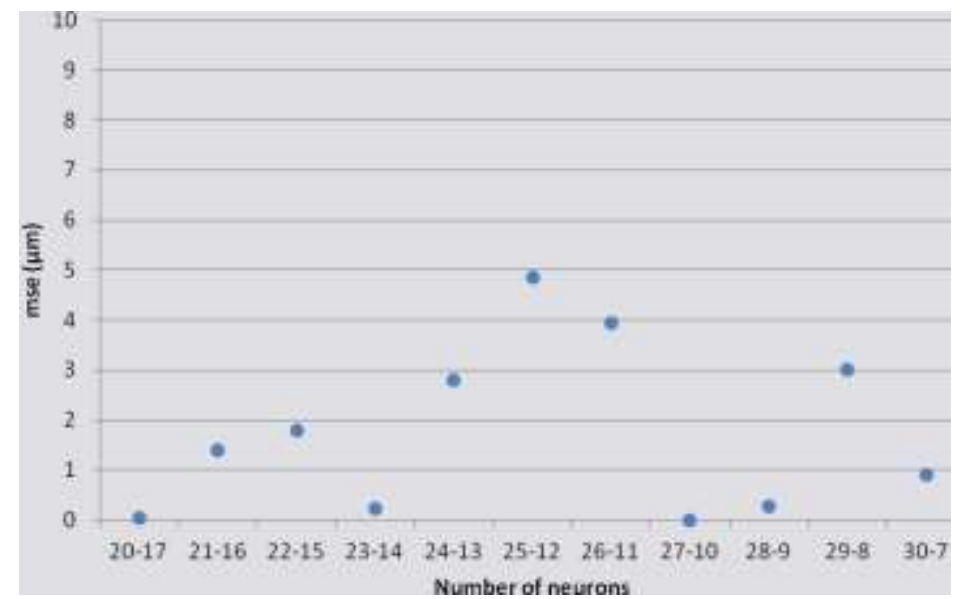

Figure 9. Mse of validation experiments vs. number of neurons, for Rk (three networks) and two hidden layers.

For three networks and two hidden layers, relatively high mse values close to 5 were found for combinations 25-12 and 26-11. Such values are higher than those reported for one network (Figure 4), below 2 in all cases. A model with 23 and 14 neurons in the first and second layer respectively was selected for Rk, since it showed lowest mse value for test experiments. A similar procedure was employed for determining number of neurons to be used for modelling parameters Rpk and Rvk. Models having 10-4 and 3-5 neurons respectively were selected. In order to compare models with one layer with models with two layers, for each one of the roughness parameters studied, mse values of test data were employed. Summary of results is presented in Table 3. 


\section{Author}

Table 3 Comparison between models for three networks.

\begin{tabular}{c|c|c}
\hline $\begin{array}{c}\text { Number of } \\
\text { layers }\end{array}$ & $\begin{array}{c}\text { Number of } \\
\text { neurons }\end{array}$ & mse \\
\hline One layer-Rk & 25 & 2.870 \\
\hline $\begin{array}{c}\text { Two layers- } \\
\text { Rk }\end{array}$ & $23-14$ & 3.577 \\
\hline $\begin{array}{c}\text { One layer- } \\
\text { Rpk }\end{array}$ & 9 & 1.042 \\
\hline $\begin{array}{c}\text { Two layers- } \\
\text { Rpk }\end{array}$ & $10-4$ & 0.767 \\
\hline $\begin{array}{c}\text { One layer- } \\
\text { Rvk }\end{array}$ & 5 & 0.911 \\
\hline $\begin{array}{c}\text { Two layers- } \\
\text { Rvk }\end{array}$ & $5-3$ & 1.234 \\
\hline
\end{tabular}

Overall results for the strategy of using three different networks are presented in Table 4, with average mse values for the three parameters, for one layer and for two layer networks respectively.

Table 4 Overall results for three networks

\begin{tabular}{c|c|c}
\hline Number of layers & $\begin{array}{c}\text { Number of } \\
\text { neurons }\end{array}$ & mse \\
\hline $\begin{array}{c}\text { One layer (average } \\
\text { Rk, Rpk and Rvk) }\end{array}$ & 25,9 and 5 & 1.608 \\
\hline $\begin{array}{c}\text { Two layers } \\
\text { (average Rk, Rpk } \\
\text { and Rvk) }\end{array}$ & $23-14,10-4$ and & 1.859 \\
\hline
\end{tabular}

Best option corresponds to one hidden layer, with lowest average mse value of 1.608 for test experiments. Hidden layer will contain 25 neurons for parameter Rk, 9 neurons for parameter Rpk and 5 neurons for parameter Rvk. 


\subsection{Comparison between one and three neural network models}

In order to compare and select the best final configuration between one network and three networks models, mean squared error of test data was calculated according to Equation 1 in Section 2.3. Table 5 contains comparison between one network and three networks, with mse values of test data for each model considered.

Table 5 Comparison between one network model and three network model

\begin{tabular}{c|c|c|c}
\hline $\begin{array}{c}\text { Number of } \\
\text { networks }\end{array}$ & $\begin{array}{c}\text { Number of } \\
\text { layers }\end{array}$ & $\begin{array}{c}\text { Number of } \\
\text { neurons }\end{array}$ & mse \\
\hline $\begin{array}{c}\text { One network } \\
\text { (average Rk, Rpk } \\
\text { and Rvk) }\end{array}$ & 2 & $23-14,10-4$ and & 1.859 \\
\hline $\begin{array}{c}\text { Three networks } \\
\left(\begin{array}{c}\text { average Rk, Rpk } \\
\text { and Rvk) }\end{array}\right.\end{array}$ & 1 & 25,9 and 5 & 1.608 \\
\hline
\end{tabular}

All selected networks have less than 30 neurons in the hidden layers. Within the range of variables studied, one hidden layer is recommended when three different networks, one for each roughness parameter, are considered. On the contrary, two hidden layers are recommended when the three roughness parameters are modelled with the same network.

According to Table 6, it can be concluded that best overall result corresponds to three networks with one hidden layer having 25,9 and 5 neurons respectively.

However, if an indirect model is to be obtained, i.e. a model in which process parameters are determined to obtain a certain roughness value, it is not possible to employ three different networks. For this reason, in this case one network would be recommended.

Simulated results for experiments 1 to 33 are presented in Table 6 for one neural network models having 26 and 11 neurons in the first and second layer respectively.

Table 6. Simulated values for Rk, Rpk and Rvk for one network having 26 and 11 neurons

\begin{tabular}{|c|c|c|c|}
\hline Experiment & $\begin{array}{c}\mathrm{Rk} \\
(\mu \mathrm{m})- \\
26-11\end{array}$ & $\begin{array}{c}\text { Rpk } \\
(\mu \mathrm{m})- \\
26-11 \\
\end{array}$ & $\begin{array}{c}\text { Rvk } \\
(\mu \mathrm{m})- \\
26-11 \\
\end{array}$ \\
\hline 1 & 10,60 & 2,82 & 1,44 \\
\hline 2 & 11,46 & 2,71 & 1,69 \\
\hline 3 & 7,80 & 2,66 & 1,79 \\
\hline
\end{tabular}




\begin{tabular}{|c|c|c|c|}
\hline 4 & 12,57 & 3,18 & 2,64 \\
\hline 5 & 12.39 & 5.99 & 5.02 \\
\hline 6 & 6,78 & 2,08 & 1,79 \\
\hline 7 & 4,04 & 2,40 & 2,24 \\
\hline 8 & 15,83 & 5,42 & 4,06 \\
\hline 9 & 6,71 & 2,40 & 1,49 \\
\hline 10 & 6,54 & 3,09 & 1,64 \\
\hline 11 & 10,32 & 2,49 & 0,54 \\
\hline 12 & 11.88 & 4.05 & 1.89 \\
\hline 13 & 8.07 & 2.36 & 1.06 \\
\hline 14 & 9,56 & 2,60 & 1,17 \\
\hline 15 & 9,09 & 4,53 & 3,18 \\
\hline 16 & 11,82 & 4,51 & 3,26 \\
\hline 17 & 10,05 & 2,44 & 2,01 \\
\hline 18 & 14,85 & 4,77 & 1,75 \\
\hline 19 & 6,18 & 2,73 & 2,44 \\
\hline 20 & 9,46 & 3,62 & 3,60 \\
\hline 21 & 7,81 & 2,51 & 2,14 \\
\hline 22 & 9,18 & 2,85 & 2,49 \\
\hline 23 & 17,86 & 6,46 & 4,70 \\
\hline 24 & 14,26 & 3,67 & 1,70 \\
\hline 25 & 7.37 & 2.72 & 0.97 \\
\hline 26 & 10,32 & 2,71 & 1,86 \\
\hline 27 & 16,44 & 5,44 & 5,04 \\
\hline 28 & 8.37 & 2.65 & 3.21 \\
\hline 29 & 17.85 & 6.48 & 3.19 \\
\hline 30 & 5.31 & 2.14 & 1.83 \\
\hline 31 & 7.92 & 3.59 & 2.58 \\
\hline
\end{tabular}


Title

\begin{tabular}{l|l|l|l}
\hline 32 & 12.65 & 5.26 & 4.20 \\
\hline 33 & 6.90 & 3.51 & 2.64 \\
\hline
\end{tabular}

For one neural network having 26 and 11 neurons in the first and second layer respectively, mse vs. epoch plot is presented in Figure 10.

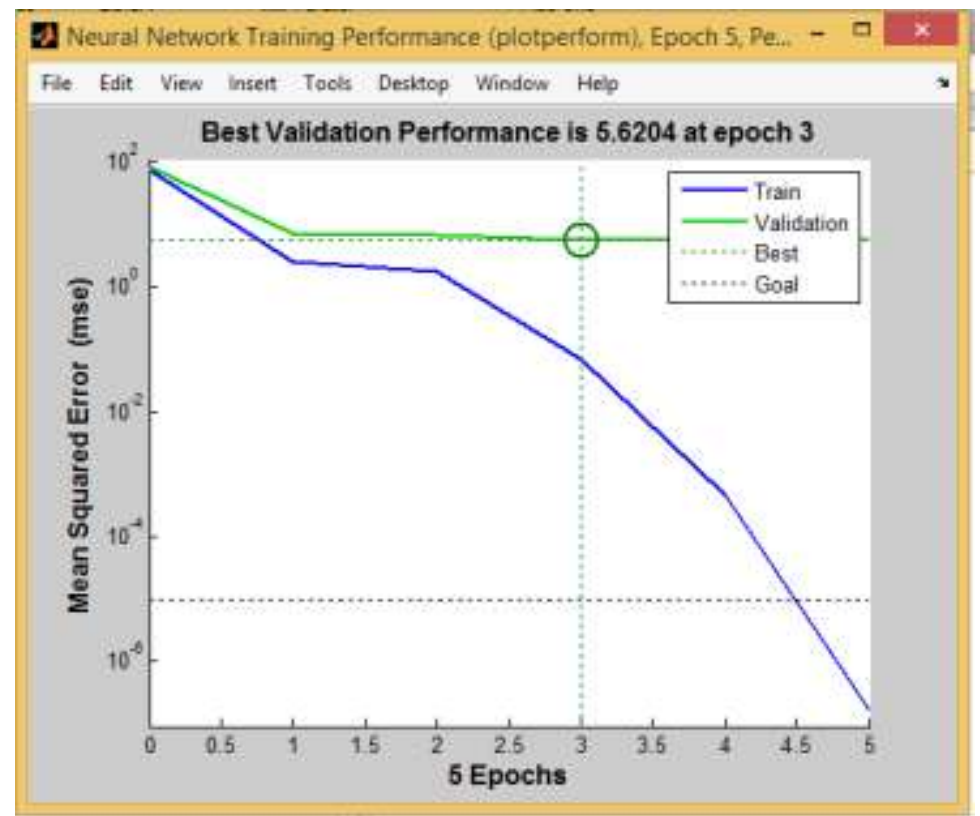

Figure 10. Mse vs. Epochs plot for one network having 26 and 11 neurons

Figure 9 depicts mean squared error (mse) of train and validation data after each training (epoch). It can be seen that with train data mean squared error decreases when training is repeated. On the contrary, for validation data (which are not used for training the network), mean squared error reaches lowest value after the third training. For this reason, training was stopped after the third epoch. Subsequent epoch would make the neural network to memorize results of training data, but performance would not improve when simulating new data.

Figure 11 shows regression fit for training, validation and overall data. 
Author

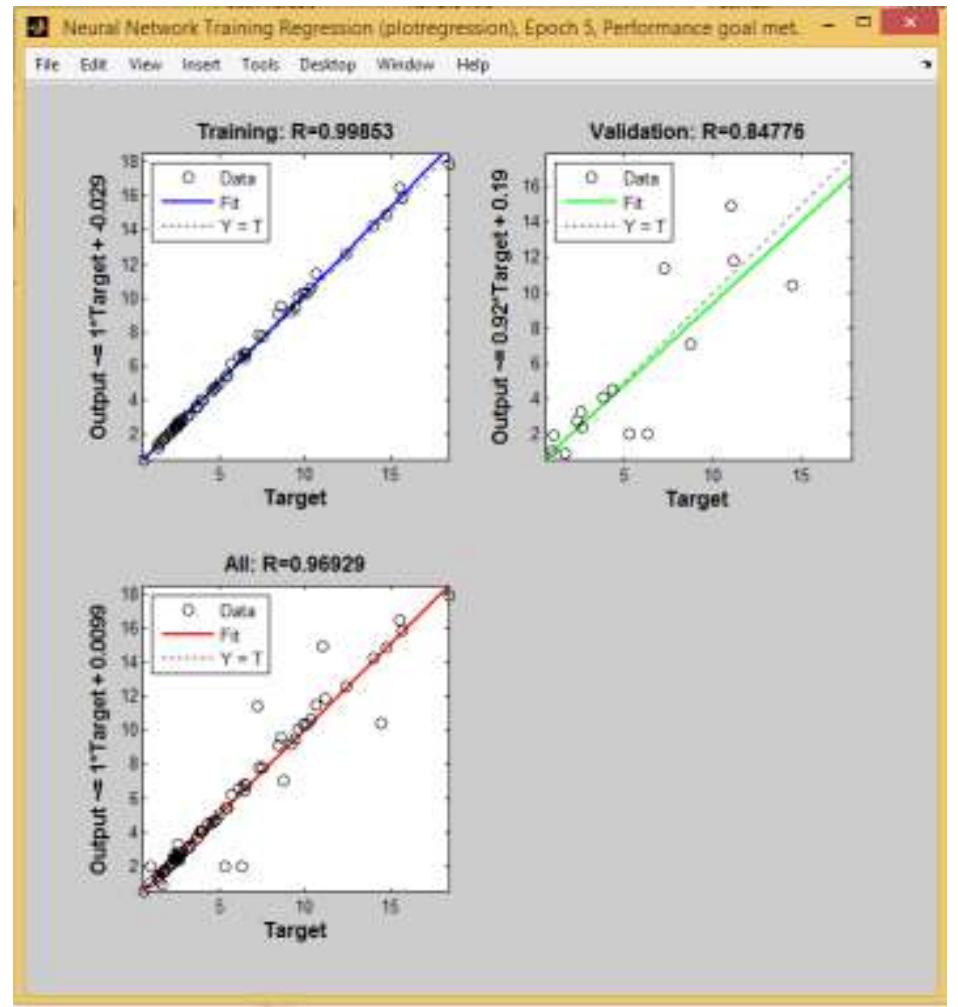

Figure 11 Regression plots for one network having 26 and 11 neurons

Fit values were higher than $84 \%$ for both training and validation data, with overall fit of $96.9 \%$.

For this reason, a one network model having 26 and 11 neurons in the first and second layer respectively is considered to be appropriate for modelling roughness parameters $\mathrm{Rk}$, Rpk and Rvk as a function of main honing process parameters.

\section{Conclusions}

Main conclusions of the paper are presented next:

- Neural networks models are appropriate for predicting roughness parameters Rk, Rpk and Rvk, corresponding to the Abbott-Firestone curve, as a function of process parameters in rough honing. Several configurations were tested for either one or three different networks, with different number of layers and different number of neurons. 
- Results demonstrated that low number of neurons below 30 is recommended in all cases, because it leads to lower mean squared error values. Number of layers depends on number of networks considered. Best overall result corresponds to use of three neural networks having one layer each, with 25, 9 and 5 neurons for $\mathrm{Rk}, \mathrm{Rpk}$ and Rvk respectively. However, use of three different networks does not allow addressing the indirect model, in which process parameters are predicted to obtain a certain roughness value. If one network is considered, best option corresponds to two layers having 26 and 11 neurons respectively.

- Models will be very helpful in industry to to predict roughness to be obtained from process parameters selected. They will also help studying the effect of each process variable separately on surface roughness, without need of experimental trial and error tests that would waste material and energy. This will improve quality of workpieces and will reduce costs.

In future research, an adaptive model will be defined to address the indirect problem, in which process parameters are selected to obtain a certain roughness value. It will combine conventional direct and indirect neural network models.

\section{References}

Aguiar, P.R. et al., 2008. Predicting Surface Roughness in Grinding Using Neural Networks. In Advances in Robotics, Automation and Control. pp. 33-44.

Bell, S.B., Maden, H. \& Needham, G., 1981. The influence of grit size and stone pressure on honing. Precision Engineering, 3(1), p.47.

Benardos, P.G. \& Vosniakos, G.C., 2002. Prediction of surface roughness in CNC face milling using neural networks and Taguchi's design of experiments. Robotics and Computer-Integrated Manufacturing, 18(5-6), pp.343-354.

Buj-Corral, I. \& Vivancos-Calvet, J., 2011. Roughness variability in the honing process of steel cylinders with CBN metal bonded tools. Precision Engineering, 35(2), pp.289-293.

Buj-Corral, I., Vivancos-Calvet, J. \& Coba-Salcedo, M., 2014. Modelling of surface finish and material removal rate in rough honing. Precision Engineering, 38(1), pp.100-108.

Chavoshi, S.Z., 2013. Modelling of surface roughness in CNC face milling of alloy stellite 6. International Journal of Computational Materials Science and Surface Engineering, 5(4), p.304.

Deepak Lawrence, K., Shanmugamani, R. \& Ramamoorthy, B., 2014. Evaluation of image based Abbott-Firestone curve parameters using machine vision for the characterization of cylinder liner surface topography. Measurement, 55, pp.318334.

Deng, Z.H. et al., 2009. A hybrid model using genetic algorithm and neural network for process parameters optimization in NC camshaft grinding. The International Journal of Advanced Manufacturing Technology, 45(9-10), pp.859-866. 
Author

Feng, C.-X., Wang, X. \& Yu, Z., 2002. Neural networks modeling of honing surface roughness parameters defined by ISO 13565. Journal of Manufacturing Systems, 21(5), pp.395-408.

Feng, C.J., Yu, Z.S. \& Kingit, U., 2005. Threefold vs . Fivefold Cross Validation in OneHidden-Layer and Two-Hidden-Layer Predictive Neural Network Modeling of Machining Surface Roughness Data. Journal of Manufacturing Systems, 24(2), pp.93-107.

Feng, Q., Ren, C. \& Pei, Z., 2015. A Physics-Based Predictive Model for Number of Contact Grains and Grain Depth of Cut in Honing. Machining Science and Technology, 19(1), pp.50-70.

FEPA, 1997. 61/97 - FEPA standard for superabrasives grain sizes.

Ben Fredj, N., Amamou, R. \& Rezgui, M.A., 2002. Surface roughness prediction based upon experimental design and neural network models. In IEEE International Conference on Systems, Man and Cybernetics. IEEE, p. 6.

ISO, 1996. ISO 13565-2:1996. Geometrical Product Specifications (GPS) -- Surface texture: Profile method; Surfaces having stratified functional properties -- Part 2: Height characterization using the linear material ratio curve. , pp.1-6.

ISO, 2005. ISO 6104:2005 - Superabrasive products -- Rotating grinding tools with diamond or cubic boron nitride -- General survey, designation and multilingual nomenclature.

Kanthababu, M., Shunmugam, M.S. \& Singaperumal, M., 2010. Data mining approach for selection of plateau honing parameters with dual objectives. International Journal of Machining and Machinability of Materials, 8(1-2), pp.167-176.

Lawrence, K.D. \& Ramamoorthy, B., 2016. Multi-surface topography targeted plateau honing for the processing of cylinder liner surfaces of automotive engines. Applied Surface Science, 365, pp.19-30.

Lei, X. et al., 2011. Method for cylindricity error evaluation using Geometry Optimization Searching Algorithm. Measurement, 44(9), pp.1556-1563.

Liao, T.W. \& Chen, L., 1994. A neural network approach for grinding processes: modelling and optimization. International Journal of Machine Tools and Manufacture, 34(7), pp.919-937.

Maksoud, T.M.A., Atia, M.R. \& Koura, M.M., 2003. Applications of Artificial Intelligence to Grinding Operations via Neural Networks. Machining Science and Technology, 7(3), pp.361-387.

Özel, T. \& Karpat, Y., 2005. Predictive modeling of surface roughness and tool wear in hard turning using regression and neural networks. International Journal of Machine Tools and Manufacture, 45(4-5), pp.467-479.

Petri, K.I., Billo, R.E. \& Biclanda, B., 1998. A Neural Network Process Model for Abrasive Flow Machining Operations. Journal of Manufacturing Systems, 17(1), pp.52-64. 
Pu, W.Y. et al., 2010. Intelligence Selection System for Honing Parameter Based on Genetics and Artificial Neural Networks. Advanced Materials Research, 102-104, pp.846-850.

Shaikh, J.H., Jain, N.K. \& Pathak, S., 2015. Performance enhancement of electrochemical honing process using ANN approach for bevel gear finishing. International Journal of Precision Technology, 5(2), p.157.

Sivatte-Adroer, M. et al., 2016. Indirect model for roughness in rough honing processes based on artificial neural networks. Precision Engineering, 43, pp.505-513.

Teimouri, R. \& Baseri, H., 2013. Artificial evolutionary approaches to produce smoother surface in magnetic abrasive finishing of hardened AISI 52100 steel. Journal of Mechanical Science and Technology, 27(2), pp.533-539.

Tung, S.C. \& McMillan, M.L., 2004. Automotive tribology overview of current advances and challenges for the future. Tribology International, 37(7), pp.517-536.

Voronov, S.A., Gouskov, A.M. \& Bobrenkov, O.A., 2009. Modelling of bore honing. International Journal of Mechatronics and Manufacturing Systems, 2(5/6), p.566. 
Author

\section{Figure captions}

Figure 1. Schematic drawing of the honing process

Figure 2. Honing test machine.

Figure 3. Flow chart for the two different strategies: a) one network, b) three networks.

Figure 4. Schematic drawing of one network with one hidden layer.

Figure 5. Roughness profiles for: a) Experiment 23, b) Experiment 9.

Figure 6. Average mse of validation experiments vs. number of neurons, for one network and one hidden layer.

Figure 7. Average mse of validation experiments vs. number of neurons, for one network and two hidden layers

Figure 8. Mse of validation experiments vs. number of neurons, for Rk (three networks) and one hidden layer.

Figure 9. Mse of validation experiments vs. number of neurons, for Rk (three networks) and two hidden layers.

Figure 10. Mse vs. Epochs plot for one network having 26 and 11 neurons

Figure 11. Mse vs. Epochs plot for one network having 26 and 11 neurons 
Title

\section{Table captions}

Table 1. Experiments performed and roughness values obtained

Table 2. Comparison between models for one network.

Table 3. Comparison between models for three networks.

Table 4. Overall results for three networks.

Table 5. Comparison between one network model and three network model

Table 6. Simulated values for Rk, Rpk and Rvk for one network having 26 and 11 neurons 